\title{
Longer Electromechanical Delay Impairs Hamstrings Explosive Force versus Quadriceps
}

\author{
RICCI HANNAH ${ }^{1,2}$, CLAIRE MINSHULL ${ }^{1}$, STEPHANIE L. SMITH ${ }^{1,3}$, and JONATHAN P. FOLLAND ${ }^{4}$ \\ ${ }^{1}$ Sport, Health and Performance Enhancement (SHAPE) Research Group, School of Science and Technology, Nottingham \\ Trent University, Nottingham, UNITED KINGDOM; ${ }^{2}$ Sobell Department of Motor Neuroscience and Movement Disorders, \\ Institute of Neurology, University College London, London, UNITED KINGDOM; ${ }^{3}$ School of Health and Life Sciences, \\ Glasgow Caledonian University, Glasgow, UNITED KINGDOM; and ${ }^{4}$ School of Sport, Exercise and Health \\ Sciences, Loughborough University, Loughborough, UNITED KINGDOM
}

\begin{abstract}
HANNAH, R., C. MINSHULL, S. L. SMITH, and J. P. FOLLAND. Longer Electromechanical Delay Impairs Hamstrings Explosive Force versus Quadriceps. Med. Sci. Sports Exerc., Vol. 46, No. 5, pp. 963-972, 2014. Introduction: Explosive neuromuscular performance refers to the ability to rapidly increase force in response to neuromuscular activation. The lower explosive force production of the hamstrings relative to the quadriceps could compromise knee joint stability and increase the risk of anterior cruciate ligament injury. However, the time course of the rise in explosive force of the hamstrings and quadriceps from their initial activation, and thus the explosive hamstringsto-quadriceps (H/Q) force ratio, has not been documented. Methods: The neuromuscular performance of 20 untrained males was assessed during a series of isometric knee flexion and extension contractions, with force and surface EMG of the hamstrings and quadriceps recorded during explosive and maximum voluntary contractions. Hamstrings force was expressed relative to quadriceps force to produce hamstring-to-quadriceps ratios of explosive H/Q force and H/Q maximum voluntary force. For the explosive contractions, agonist electromechanical delay (EMD), agonist and antagonist neural activation were assessed. Results: The quadriceps was $79 \%$ stronger than the hamstrings, but quadriceps explosive force was up to $480 \%$ greater than the hamstrings from 25 to $50 \mathrm{~ms}$ after first activation. Consequently, the explosive $\mathrm{H} / \mathrm{Q}$ force ratio was very low at 25 and $50 \mathrm{~ms}(0 \%-17 \%)$ and significantly different from H/Q maximum voluntary force ratio (56\%). Hamstrings EMD was 95\% greater than quadriceps EMD (44.0 vs $22.6 \mathrm{~ms})$, resulting in a $21-\mathrm{ms}$ later onset of force in the hamstrings that appeared to explain the low explosive H/Q force ratio in the early phase of activation. Conclusions: Prolonged hamstrings EMD appears to impair early phase $(0-50 \mathrm{~ms})$ explosive force production relative to the quadriceps and may render the knee unstable and prone to anterior cruciate ligament injury during this period. Key Words: KNEE JOINT, MUSCLE STRENGTH, ELECTROMYOGRAPHY, RATE OF FORCE DEVELOPMENT, ANTERIOR CRUCIATE LIGAMENT
\end{abstract}

$\mathrm{T}$ he hamstrings-to-quadriceps $(\mathrm{H} / \mathrm{Q})$ ratio of maximum voluntary force (MVF) or torque is commonly used in the sports medicine literature to describe the reciprocal muscle function about the knee joint $(2,3,8,14,24)$ and is thought to provide a measure of the capacity for muscular stabilization of the knee $(2,3)$. For example, the anterior cruciate ligament (ACL) is the primary ligamentous restraint to anterior tibial translation (11). Force produced

Address for correspondence: Ricci Hannah, Ph.D., Institute of Neurology, University College London, Box 146, Queen Square, London WC1 3BG, United Kingdom; E-mail: r.hannah@ucl.ac.uk.

Submitted for publication March 2013.

Accepted for publication October 2013

0195-9131/14/4605-0963/0

MEDICINE \& SCIENCE IN SPORTS \& EXERCISE EX Copyright (C) 2014 by the American College of Sports Medicine

DOI: 10.1249/MSS.0000000000000188 by quadriceps muscle contraction elicits anterior tibial translation, particularly with the knee close to full extension, and thus can contribute to the loading (37) and rupture of the ACL (17). In contrast, the contraction of the hamstrings may protect the ACL by opposing anterior tibial shear (37). Thus, the H/Q MVF ratio may provide a measure of the hamstrings' ability to resist the anterior shear produced by quadriceps contraction $(2,3)$, with a low H/Q MVF ratio being indicative of a reduced capacity for dynamic knee joint stabilization $(2,3)$.

A low H/Q MVF ratio has been suggested to increase the risk of noncontact ACL injury (20,34). However, noncontact ACL injuries sustained during athletic movements, for example, jump landings, are estimated to occur within $50 \mathrm{~ms}$ of foot contact with the ground (26). In contrast, the time taken to attain MVF during an isometric contraction is typically $>300 \mathrm{~ms}$ (41). The discrepancy in the time course of these events may confound any association 
between the H/Q MVF ratio and the injury risk. Because MVF seems unlikely to be attained in the time available, the capacity for knee joint stabilization may depend more on the relative ability of the knee muscles to rapidly exert force during the initial rising phase of contraction, and particularly during the early phase (first $50 \mathrm{~ms}$ ) after neuromuscular activation.

Explosive neuromuscular performance can be defined as the ability to rapidly increase force in response to neuromuscular activation and can be quantified as the force produced at specific time points from activation onset throughout the rising phase of force production. The explosive neuromuscular performance of the hamstrings relative to the quadriceps, quantitatively assessed by the explosive $\mathrm{H} / \mathrm{Q}$ force ratio, may better reflect the capacity for rapid muscular stabilization of the knee compared with the H/Q MVF ratio. Therefore, further research is required to understand the time course of the rise in explosive force of the hamstrings and quadriceps and, thus, the explosive H/Q force ratio.

Two important determinants of explosive neuromuscular performance are the electromechanical delay (EMD) and the rate of force development, which are defined as the time difference between the onset of EMG activity and the onset of force (12) and the rapidity by which the force increases after onset $(1,16,42)$, respectively. Although these parameters have often been studied as distinct phenomena $(1,16,21,28,42)$, both are integral to rapid force production and joint stabilization. Data from separate studies in our laboratory suggest that the EMD of the hamstrings $(28,29)$ is approximately twice as long as EMD of the quadriceps $(21,30)$, with values of approximately 50 versus $26 \mathrm{~ms}$. Assuming the simultaneous activation of the knee joint muscles in response to an injury-related threat, this longer EMD of the hamstrings would be expected to delay the production of knee flexion force relative to extension force. If this were the case, the explosive H/Q force ratio during the early phase of activation would be very low, potentially substantially lower than the H/Q MVF ratio, and this could make the knee joint inherently unstable during the early phase of activation. Therefore, we hypothesized that the hamstrings would have a longer EMD than the quadriceps, which would compromise explosive H/Q force ratio during the early phase of activation.

We have previously documented much greater interindividual variation in knee extensor explosive force compared with MVF (18), but the variability in the explosive $\mathrm{H} / \mathrm{Q}$ force ratio and how this compares with the H/Q MVF ratio is unknown. The extent of interindividual variation in the explosive H/Q force ratio may reflect the variable capacity for knee joint stabilization and injury risk. Furthermore, understanding the mechanisms for the explosive $H / Q$ force ratio may aid in the development of training strategies to enhance reciprocal muscle function and dynamic joint stability. In addition to EMD, the explosive H/Q force ratio may depend on the ability to explosively express the available force generating capacity of each muscle, that is, explosive force normalized to MVF, which may in turn depend on agonist neuromuscular activation, as this is known to influence the rate of force production $(1,16,18,42)$. However, the contribution of these mechanisms to the explosive H/Q force ratio has not been considered.

The purpose of this study was to compare the explosive neuromuscular performance of the hamstrings and quadriceps and to contrast the explosive H/Q force ratio with the traditionally reported $\mathrm{H} / \mathrm{Q} \mathrm{MVF}$ ratio. The mechanisms for the explosive performance of the opposing muscle groups, and thus the explosive H/Q force ratio, were investigated by comparing EMD, subsequent explosive force production in absolute terms and normalized to MVF, and neuromuscular activation with EMG. Furthermore, these comparisons were made with the knee close to full extension $\left(150^{\circ}\right)$, which reflects the position of the knee where the greatest strain on the ACL occurs (37), when the foot contacts the ground during athletic locomotor movements $(19,27)$ and a position where the majority of ACL injuries occur (26). Because differences between individuals in explosive H/Q force ratio may help to explain differences in their capacity for knee joint stabilization, an additional aim of the study was to document the magnitude of interindividual variability in explosive $\mathrm{H} / \mathrm{Q}$ force ratio.

\section{METHODS}

\section{Participants}

Twenty untrained males were recruited, and their characteristics were as follows (mean $\pm \mathrm{SD}$ ): age $=25 \pm 5 \mathrm{yr}$, height $=179 \pm 5 \mathrm{~cm}$, body mass $=76.5 \pm 9.8 \mathrm{~kg}$, and body mass index $=23.8 \pm 3.2 \mathrm{~kg} \cdot \mathrm{m}^{-2}$. Participants were categorized using the International Physical Activity Questionnaire Short Format (http://www.ipaq.ki.se/index.html [13]) as having moderate habitual levels of physical activity with a score of $1891 \pm 966 \mathrm{MET} \cdot \mathrm{min} \cdot \mathrm{wk}^{-1}$. Participants performed vigorous activity such as playing soccer or fast cycling $1 \pm 1 \mathrm{~d} \cdot \mathrm{wk}^{-1}$ for $40 \pm 43 \mathrm{~min}$ and moderate activity such as doubles tennis or regular paced cycling on $2 \pm 2 \mathrm{~d} \cdot \mathrm{wk}^{-1}$ for $45 \pm 36 \mathrm{~min}$. To minimize the potentially confounding effects of training background on neuromuscular performance and particularly H/Q ratios, exclusion criteria included any history of systematic strength, power or aerobic training. All participants were healthy and free from musculoskeletal injury to the lower limbs. Volunteers provided written informed consent before their participation in this study, which was approved by the University Human Ethical Review Committee.

\section{Study Design}

Participants visited the laboratory for $60 \mathrm{~min}$ on two separate occasions to complete a familiarization and one test session. All sessions commenced between 10:00 a.m. and 4:00 p.m. The familiarization and test sessions were separated by $1 \mathrm{wk}$, and both sessions consisted of the same protocol. 

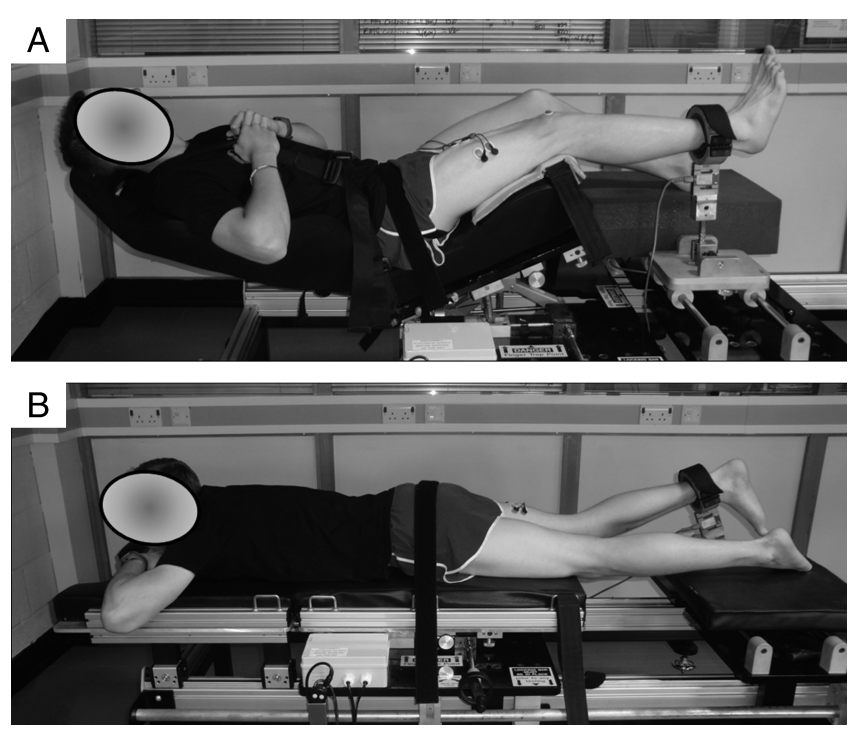

FIGURE 1-Participant orientation during assessments of knee extension (A) and flexion (B) force.

Participants were positioned in custom-built dynamometers to obtain measures of isometric hamstrings and quadriceps performance of the dominant limb. The order of hamstrings and quadriceps testing was randomized. A series of maximum voluntary contractions (MVC) and explosive voluntary contractions were completed in that order in each dynamometer.

\section{Measurements}

Knee extension force. Participants were seated supine in a custom built dynamometer $(21,30)$ with hip and knee joint angles of $140^{\circ}$ and $150^{\circ}\left(180^{\circ}=\right.$ full extension; Fig. 1A), respectively. Adjustable strapping across the pelvis and shoulders prevented extraneous movement during muscle activation. An ankle cuff was attached to the dominant leg of the participant $\sim 3 \mathrm{~cm}$ proximal to the lateral malleolus and was in series with a linear strain gauge (615; Tedea-Huntleigh, Herzliya, Israel) oriented perpendicular to the tibia. The force signal was amplified $(\times 1000)$ and sampled at $2000 \mathrm{~Hz}$ using an external A/D converter (1401; CED, Cambridge, UK), interfaced with a PC using Spike 2 software (CED).

Knee flexion force. Participants were secured in a prone position on a custom-built dynamometer $(28,29)$, with hip and knee joint angles of $180^{\circ}$ and $150^{\circ}$ (Fig. 1B), respectively. Adjustable strapping across the pelvis prevented extraneous movement during muscle activation. The same ankle cuff and strain gauge used for knee extension force measurements were attached to the dominant leg of the participant $\sim 3 \mathrm{~cm}$ proximal to the lateral malleolus and oriented perpendicular to the tibia. The force signal was sampled in the same manner as knee extension force.

The hardware and software for assessments of knee flexion and extension force were the same, and the use of two dynamometers ensured that agonist muscle EMG was not subject to compression or movement artifacts. For instance, performing knee flexion contractions while in a seated position could affect hamstring EMG by virtue of the electrodes being between the hamstrings and the seat. Individual positioning for the prescribed joint configurations was established during the familiarization session while the participant performed submaximal voluntary contractions to $\sim 50 \%$ MVF. The characteristics of the dynamometers ensured minimal extraneous movement and changes in knee angles during contraction. In pilot experiments with five participants, an electrogoniometer (SG150, Biometrics Ltd., Gwent, UK; preamplified lead, Noraxon, Scottsdale, USA) was attached to either side of the lateral aspect of the knee and used to record changes in knee joint angle during flexion and extension contractions. Signals were sampled at $2000 \mathrm{~Hz}$ and acquired into Spike 2 simultaneously with the force signals via the same A/D converter. Small changes in knee joint angle of $4^{\circ} \pm 1^{\circ}$ from rest to MVF were found with both dynamometers, which is considerably less than the movement afforded by some commercially available dynamometers $\left(>15^{\circ}\right)(46)$.

EMG. EMG signals were recorded from the superficial quadriceps and hamstrings: rectus femoris, vastus medialis, vastus lateralis, biceps femoris long head, and semitendinosus. After preparation of the skin by shaving, light abrasion, and cleaning with alcohol, bipolar surface electrodes $(3 \mathrm{~cm}$ interelectrode distance; silver/silver chloride, $95 \mathrm{~mm}^{2}$ area; Ambu Blue Sensor, Ambu, Ballerup, Denmark) were attached over each muscle at standardized percentages of thigh length measured from the knee joint space to greater trochanter: rectus femoris, $60 \%$; vastus lateralis, biceps femoris long head, and semitendinosus, $40 \%$; vastus medialis, $20 \%$. Electrodes were positioned parallel to the presumed orientation of the muscle fibers. EMG signals were preamplified by active EMG leads (input impedance $100 \mathrm{M} \Omega, \mathrm{CMMR}>100 \mathrm{~dB}$, base gain 500 , first-order high-pass filter set to $10 \mathrm{~Hz}$; Noraxon, Scottsdale, AZ) connected in series to a custom-built junction box and subsequently to the same A/D converter and PC software that enabled synchronization with the force data. The signals were sampled at $2000 \mathrm{~Hz}$. EMG data were band-pass filtered in both directions between 20 and $450 \mathrm{~Hz}$ using a fourth-order zero-lag Butterworth filter before analysis.

\section{Protocol}

Maximum voluntary knee extension and flexion contractions. Participants performed a series of warm-up contractions at $\sim 50 \%, 75 \%$, and $90 \%$ of MVF, before completing a series of three MVC. Warm-up and MVC lasted $\sim 3 \mathrm{~s}$ and were preceded by $\sim 30 \mathrm{~s}$ rest. For the MVC, participants were instructed to contract as hard as possible for $3 \mathrm{~s}$. They received strong verbal encouragement during the contractions and real-time biofeedback regarding the force response during and after each contraction.

MVF of the quadriceps (QMVF) and hamstrings (HMVF) was defined as the greatest instantaneous force produced during the relevant series of contractions and expressed in absolute terms (N). The H/Q MVF ratio was calculated as HMVF divided by QMVF and expressed as a percentage (\%). The maximal EMG amplitude of the agonist muscles 
during knee extension and flexion MVC was calculated as the root mean square of a 500-ms epoch surrounding MVF (10), $250 \mathrm{~ms}$ either side. Agonist EMG values were averaged to calculate mean quadriceps $\left(\mathrm{QEMG}_{\max }\right)$ and hamstrings $\left(\mathrm{HEMG}_{\max }\right)$ values. Antagonist muscle coactivation extension and flexion MVC was recorded during the same $500 \mathrm{~ms}$ epoch and expressed as a percentage of the respective $\mathrm{EMG}_{\max }$ of each muscle, before calculating mean quadriceps and hamstrings values, respectively.

Explosive voluntary knee extension and flexion contractions. Measurements on each dynamometer involved participants completing a series of 10 explosive isometric contractions, each separated by a 20 -s rest. Participants were instructed to extend or flex their knee "as fast and hard as possible" for $1 \mathrm{~s}$ upon hearing an auditory signal, with the emphasis on "fast." To provide biofeedback on their explosive performance, the slope of the force-time curve with a 2-ms time constant was displayed throughout the contractions, with the peak slope (maximum RFD) of their best attempt highlighted with an on-screen cursor. Strong verbal encouragement was provided to participants to exceed this target during each subsequent contraction. A visual marker on the screen depicted $80 \%$ of MVF, which participants were expected to achieve or exceed during each explosive contraction. The resting force was also displayed on a sensitive scale during all explosive contractions to aid the detection of pretension or countermovement. The explosive contractions were performed until 10 contractions with no prior countermovement or pretension been had been recorded.

The three contractions with the greatest maximum RFD and no prior countermovement or pretension were used for analysis. Analyses involved measurement of the force-time and EMG-time traces in short periods after their onsets. Force and EMG onsets were identified visually $(4,22,39)$ by the same investigator and in accordance with a previously published method (42), which is considered to be more valid than automated methods (44). The baseline noise of the EMG signals was $0.002 \mathrm{mV}$, equivalent to $0.6 \%$ of $\mathrm{EMG}_{\max }$. The baseline noise of the force signal, calculated as the standard deviation for $1 \mathrm{~s}$, was $0.12 \mathrm{~N}$. These low levels of noise illustrate the high quality of signal recording. Force and EMG recordings were initially viewed with consistent $y$-axis scales of $5 \mathrm{~N}$ and $0.05 \mathrm{mV}$ and an $x$-axis scale of $500 \mathrm{~ms}$. These scales provided an appropriate viewing window for the accurate visual discrimination of signal onset, which was defined as the last trough before the signal deflected away from baseline noise. A vertical cursor was then manually placed on the signal onset before the signals were viewed in a magnified viewing window ( $1 \mathrm{~N}$ and $0.02 \mathrm{mV}$ for force and EMG and an $x$-axis of $25 \mathrm{~ms}$ ) to verify the accurate placement of the cursor. The slope of the force-time trace was also displayed to confirm the instant of force onset.

The time difference between the onset of EMG and force (EMD) was determined for each of the agonist muscles, and the longest EMD of the agonist muscles for each contraction

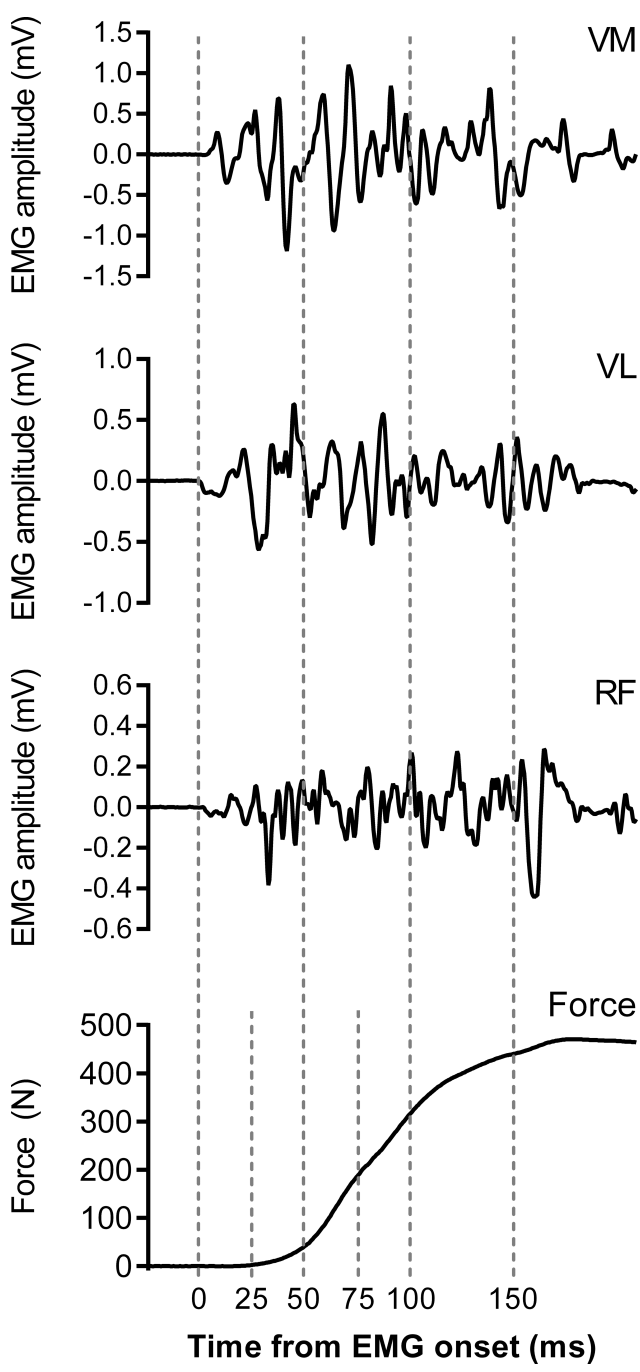

FIGURE 2-Example quadriceps EMG and knee extension force recordings for one participant during the initial $200 \mathrm{~ms}$ from first activation of an agonist muscle. EMG and force signals were analyzed in short time intervals from the EMG onset of the first agonist muscle to become active - in this instance, the vastus lateralis (VL).

was described as $\mathrm{EMD}_{\max }$ (i.e., $\mathrm{HEMD}_{\max }$ during knee flexion and $\mathrm{QEMD}_{\max }$ for knee extension). $\mathrm{EMD}_{\max }$ was expressed in absolute terms for each muscle group (ms).

Explosive force was measured at 25, 50, 75, 100, and $150 \mathrm{~ms}$ from the EMG onset of the first agonist muscle to become active (Fig. 2) and expressed in absolute terms, normalized to MVF (\%) and as a ratio of $\mathrm{H} / \mathrm{Q}$ force (explosive $\mathrm{H} / \mathrm{Q}$ force; \%) at each respective time point. The root mean square of the EMG signal for each of the five muscles was calculated over sequential 50-ms periods from EMG onset $\left(\mathrm{EMG}_{0-50}, \mathrm{EMG}_{50-100}\right.$, and $\mathrm{EMG}_{100-150}$; Fig. 2), that is, first agonist muscle to be activated. EMG values were then expressed as a percentage of the $\mathrm{EMG}_{\max }(10)$ before calculating mean quadriceps (QEMG) and hamstrings (HEMG) values. All force and EMG measurements were averaged across the three contractions selected for analysis.

As a follow-up analysis, to examine explosive force production after, and thus independently from EMD, force 
during explosive knee extension and flexion contractions was also measured at 25, 50, 75, 100 and $150 \mathrm{~ms}$ from the instant of force onset. Force was expressed in absolute terms and normalized to MVF (\%MVF). Force during explosive knee flexion contractions was also expressed as a percentage of explosive knee extension force at each time point to produce another measure of explosive $\mathrm{H} / \mathrm{Q}$ force $(\%)$.

Statistical analyses. Descriptive and outcome statistics are presented as mean \pm SD. The interindividual coefficient of variation (CV) calculated as (group SD / group mean) $\times 100$ was used to describe the between participant variability. Percentage differences in indices of hamstrings and quadriceps performance were calculated using group mean data. For indices measured at two or more time points such as EMG and explosive force, the effects of muscle group and time were analyzed using a two-way repeated-measures ANOVA (muscle group $\times$ time). A one-way repeated-measures ANOVA was used to detect differences in explosive $\mathrm{H} / \mathrm{Q}$ force over time, including H/Q MVF as the final time point because this is the ultimate end point for explosive $\mathrm{H} / \mathrm{Q}$ force production. When a significant interaction (two-way ANOVA) or effect of time (one-way ANOVA) was detected, pairwise comparisons with a Bonferroni correction were performed to determine where specific differences lay, that is, $P$ values obtained from statistical analyses were multiplied by $n$ comparisons and compared with the critical $P$ value of 0.05 . All other comparisons between muscle groups were assessed using a paired-samples $t$-test. Pearson's correlations were used to assess the relationship of the explosive $\mathrm{H} / \mathrm{Q}$ force ratio at $50 \mathrm{~ms}$ from EMG onset with $\mathrm{HEMD}_{\max }$ and the difference between $\mathrm{QEMD}_{\max }$ and $\mathrm{HEMD}_{\max }$. At $25 \mathrm{~ms}$ from EMG onset knee flexion force was equal to $0 \mathrm{~N}$; therefore, the percentage difference in knee flexion and extension force as well as the CV for knee flexion force could not be calculated. Statistical tests were all performed using the Statistical Package for the Social Sciences (version 19; SPSS Inc., Chicago, IL), and statistical significance was set at $P<0.05$.

\section{RESULTS}

Maximum voluntary force of the quadriceps and hamstrings. QMVF was 79\% higher than HMVF (586 \pm 80 vs $328 \pm 66 \mathrm{~N}$; Fig. 3A). The ratio of H/Q MVF was therefore $56 \% \pm 6 \%$ (Fig. 3B).

Explosive performance of the quadriceps and hamstrings. During the explosive contractions, absolute quadriceps force was substantially greater than hamstrings force at all time points from 25 to $150 \mathrm{~ms}$ after EMG onset (all $P<0.001$; Fig. 3A). This difference was particularly marked during the early phase of activation $(25-50 \mathrm{~ms})$, when quadriceps force was more than five times greater than hamstrings force. Consequently, the explosive $\mathrm{H} / \mathrm{Q}$ force ratio displayed a distinct time course (Fig. 3B), being very low during the early phase of activation, rising from $0 \% \pm$ $0 \%$ at $25 \mathrm{~ms}$ to $17 \% \pm 16 \%$ at $50 \mathrm{~ms}$ and, therefore, significantly less than H/Q MVF $(56 \% \pm 6 \%, P<0.001)$ during
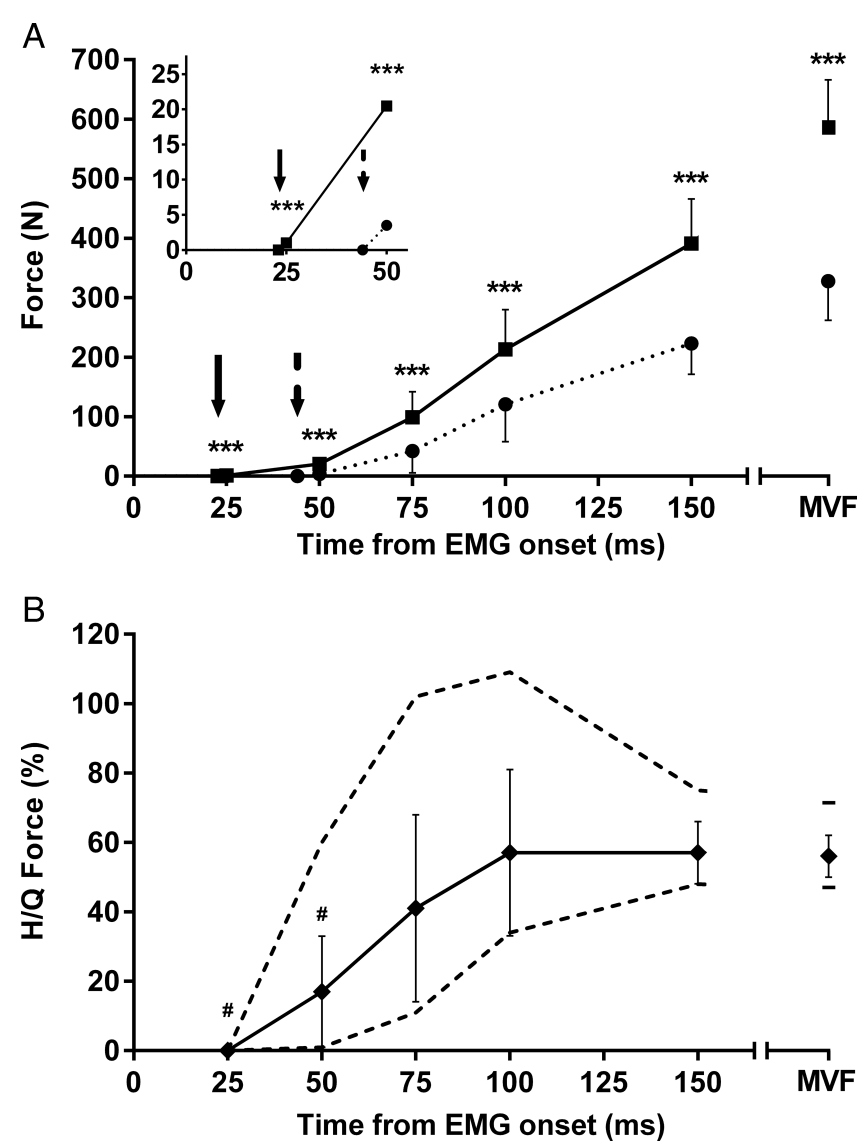

FIGURE 3-A. Absolute explosive and maximum voluntary force (MVF) of the hamstrings (closed circles) and quadriceps (closed squares). $B$. The explosive and MVF $\mathrm{H} / \mathrm{Q}$ ratios. The inset in panel $\mathrm{A}$ is a magnification of the first $50 \mathrm{~ms}$ of muscle activation, and the arrows and first data points indicate the onset of extension (solid arrow) and knee flexion (dashed arrow) force and thus the duration of EMD. Dashed lines represent the minimum and maximum values for individuals within the group (B). Data are mean \pm SD. $* * * P<\mathbf{0 . 0 0 1}$, difference between muscle groups; ${ }^{\#} P<0.001$, difference compared with H/Q MVF.

this period. The explosive $\mathrm{H} / \mathrm{Q}$ force ratio continued to rise and was statistically similar to H/Q MVF from $75 \mathrm{~ms}$ onward $(41 \% \pm 27 \%$ at $75 \mathrm{~ms}, 57 \% \pm 24 \%$ at $100 \mathrm{~ms}$, and $57 \% \pm 9 \%$ at $150 \mathrm{~ms}$; Fig. 3B).

$\mathrm{HEMD}_{\text {max }}$ was almost two times longer than $\mathrm{QEMD}_{\text {max }}$, $44.0 \pm 7.5$ versus $22.6 \pm 2.9 \mathrm{~ms}(P<0.001$; Fig. $3 \mathrm{~A}$, inset $)$. Hence, $\mathrm{HEMD}_{\max }$ was $21.4 \pm 7.3 \mathrm{~ms}(+93 \%$; range $=10-38 \mathrm{~ms})$ longer than QEMD $_{\max }$. Explosive H/Q force $50 \mathrm{~ms}$ after EMG onset was strongly negatively related to $\mathrm{HEMD}_{\max }(R=-0.83$, $P<0.0001$; Fig. 4) and the difference between $\mathrm{QEMD}_{\max }$ and $\mathrm{HEMD}_{\max }(R=-0.71, P<0.001)$.

During the explosive knee extension and flexion contractions, agonist muscle EMG amplitudes were submaximal initially $\left(\mathrm{HEMG}_{0-50},=51 \% \pm 21 \% \mathrm{HEMG}_{\max } ; \mathrm{QEMG}_{0-50}=\right.$ $53 \% \pm 27 \% \mathrm{QEMG}_{\max }$; Fig. 5) but were generally high or maximal from then on (e.g., $\mathrm{HEMG}_{50-100}=91 \% \pm 21 \%$ $\mathrm{HEMG}_{\max } ; \mathrm{QEMG}_{50-100}=99 \% \pm 24 \% \mathrm{QEMG}_{\max }$; Fig. 4). There was no main effect of muscle group on agonist EMG (muscle group, $P=0.79$ ), but there was a muscle grouptime interaction $(P=0.006)$, and post hoc tests revealed 


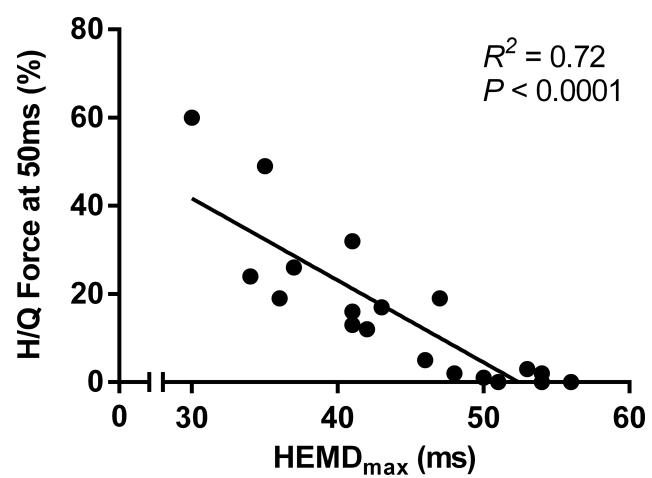

FIGURE 4-Relationship between hamstrings EMD (HEMD (Hax $_{\text {) and }}$ the explosive $\mathrm{H} / \mathrm{Q}$ force ratio $50 \mathrm{~ms}$ after $\mathrm{EMG}$ onset.

that agonist hamstrings EMG was $15 \%$ greater than agonist quadriceps EMG in the 100- to 150 -ms period, but not the 0 - to 50 -ms or 50 - to $100-\mathrm{ms}$ periods. There was no significant interaction (muscle group-time interaction, $P=0.543$ ) or main effect of muscle group $(P=0.055)$ for antagonist EMG during explosive knee extension $\left(\% \mathrm{HEMG}_{\max }: 0-50 \mathrm{~ms}\right.$, $5 \% \pm 3 \% ; 50-100 \mathrm{~ms}, 9 \% \pm 4 \% ; 100-150 \mathrm{~ms}, 9 \% \pm 4 \%)$ and flexion contractions $\left(\% \mathrm{QEMG}_{\max }: 0-50 \mathrm{~ms}, 3 \% \pm 2 \%\right.$; $50-100 \mathrm{~ms}, 7 \% \pm 2 \% ; 100-150 \mathrm{~ms}, 7 \% \pm 2 \%$ ).

By comparison with the interindividual variability $(\mathrm{CV} ; \%)$ in H/Q MVF (11\%), the variability in explosive H/Q force was much greater during the early phase of activation but decreased to a similar low level as the activation progressed (50 ms, 98\%; $75 \mathrm{~ms}, 67 \%$; $100 \mathrm{~ms}, 42 \%$; and $150 \mathrm{~ms}, 15 \%$ ). Notably, the variability (CV) in knee flexion force was 2.4 times greater by comparison with the quadriceps in the early phase of activation (50 ms, $136 \%$ vs $57 \%$ ) but was similar at $150 \mathrm{~ms}(23 \%$ vs $19 \%)$ and for MVF (20\% vs $14 \%)$.

Explosive voluntary force of the quadriceps and hamstrings independent of EMD. When the influence of EMD was removed by comparing the explosive force production of the quadriceps and hamstrings from their respective force onsets, a very different pattern was revealed compared with explosive force measured from EMG onset. There was a significant muscle group by time interaction for explosive force $(P<0.001)$. Post hoc tests indicated that absolute hamstrings force was greater than quadriceps force at $25 \mathrm{~ms}$ from force onset $(+39 \%, P=0.007$; Fig. 6A), the two muscle groups were similar at $50 \mathrm{~ms}$, but hamstrings were lower from $75 \mathrm{~ms}$ onward $(75 \mathrm{~ms},-14 \% ; 100 \mathrm{~ms}$, $-29 \%$; $150 \mathrm{~ms},-39 \%$; all time points, $P<0.001)$. Therefore, when measured from force onset, explosive H/Q force exceeded $100 \%$ in the early phase of contraction (e.g., $25 \mathrm{~ms}, 144 \% \pm 56 \%$; $50 \mathrm{~ms}, 117 \% \pm 40 \%$ ) but declined to values approaching H/Q MVF during the later phase of explosive contraction (150 ms, 61\% $\pm 7 \%$; Fig. 6C). Explosive $\mathrm{H} / \mathrm{Q}$ force measured from force onset was significantly greater than $\mathrm{H} / \mathrm{Q} \mathrm{MVF}$ at all time points up to $150 \mathrm{~ms}$ (time, $P<0.001$; all time points, $P<0.05$; Fig. $6 \mathrm{C}$ ). When explosive force was normalized to MVF of each muscle group (Fig. 6B), the hamstrings achieved a greater proportion of MVF than the quadriceps throughout the explosive contraction (muscle group $\times$ time, $P<0.001 ; 25 \mathrm{~ms},+139 \%$; $50 \mathrm{~ms},+97 \%$; $75 \mathrm{~ms},+54 \%$; $100 \mathrm{~ms},+26 \%$; $150 \mathrm{~ms},+9 \%$; all time points, $P<0.001$ except $150 \mathrm{~ms}$ where $P=0.021$ ).

\section{DISCUSSION}

The present study compared the explosive neuromuscular performance of the hamstrings and quadriceps from their initial activation. The main finding was that during the early phase of explosive contraction (0-50 ms after EMG onset), the explosive H/Q force ratio was very low $(0 \%-17 \%)$ and substantially less than the more commonly reported ratio H/Q MVF (56\%). The production of hamstrings force in relation to quadriceps force is considered critical to preventing anterior tibial translation and protecting the ACL from injury $(2,3,20,34)$. Therefore, this low early ratio of explosive H/Q force may render the knee particularly unstable and prone to anterior tibial translation in the early phase of contraction when ACL injuries are thought to occur (26). Furthermore, hamstrings EMD was approximately twice as long as quadriceps EMD, resulting in a 21-ms later onset of force in the hamstrings that appeared to explain the low explosive H/Q force ratio in the early phase of activation.

The H/Q MVF ratio. The relative strength of the hamstrings versus quadriceps, commonly described by the H/Q MVF or maximal voluntary torque ratio $(2,3,8,14,24)$, was $56 \%$ in the current study, indicating that quadriceps strength was $79 \%$ greater than hamstrings strength. This likely reflects, to a large degree, differences in muscle morphology because the physiological cross-sectional area of the quadriceps is $\sim 93 \%$ greater than that of the hamstrings (47). Although the H/Q MVF ratio has been suggested as an indicator of knee joint stability and injury risk $(2,3,20,34)$, given the short time available for knee joint stabilization during athletic locomotor tasks (26) and the relatively long time taken to achieve MVF (41), the ratio of explosive H/Q force may better reflect the capacity for rapid muscular stabilization of the knee.

Explosive performance of the hamstrings versus quadriceps. As would be expected given the differences in $\mathrm{MVF}$, during the explosive contractions, the hamstrings

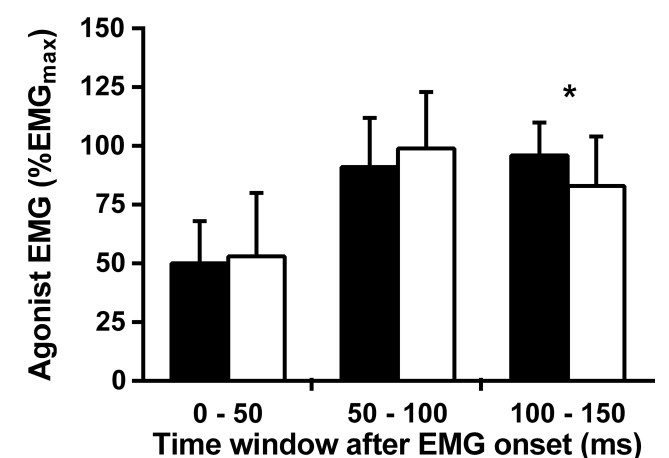

FIGURE 5-Normalized agonist hamstrings (filled bars) and quadriceps (open bars) EMG amplitude during each 50-ms period of the explosive voluntary contractions. Data are mean \pm SD. $* P<0.05$, difference between muscle groups. 
achieved less absolute force than the quadriceps at all time points from agonist EMG onset. However, the magnitude of this difference between the muscle groups during the early phase of activation was much larger than would be expected based on their respective MVF values, for example, after $50 \mathrm{~ms}$ of activation quadriceps force was $480 \%$ higher than that of the hamstrings. This resulted in a much lower explosive $\mathrm{H} / \mathrm{Q}$ force ratio at $25(0 \%)$ and $50 \mathrm{~ms}(17 \%)$ than the ratio of H/Q MVF (56\%). This low explosive H/Q force ratio during the early phase of activation has not previously been documented and may account for the apparent susceptibility for ACL injury during the initial $50 \mathrm{~ms}$ of landing (26).

This study evaluated explosive neuromuscular performance incorporating EMD because the neuromuscular response to a particular stimulus, such as an injury related threat, inherently involves an EMD. The explosive H/Q force ratio was lower than H/Q MVF in the first $50 \mathrm{~ms}$ when measured from EMG onset (Fig. 3B) but greater throughout the first $150 \mathrm{~ms}$ when measured from force onset (Fig. 6C), demonstrating the importance of incorporating EMD into evaluations of explosive neuromuscular performance and specifically the explosive $\mathrm{H} / \mathrm{Q}$ force ratio. A previous study measured explosive H/Q force from force rather than EMG onset. In contrast to the current findings, they reported a low ratio in explosive $\mathrm{H} / \mathrm{Q}$ force in the first $50 \mathrm{~ms}$ from force onset in comparison with $\mathrm{H} / \mathrm{Q}$ MVF (50). This discrepancy could be due to a range of methodological differences. Zebis et al. (50) used a commercially available dynamometer, which have been demonstrated to afford considerable compliance during "isometric" contractions (46), used an automated threshold for detecting force onset that excludes the first $\sim 25 \mathrm{~ms}$ of contraction (44) and recorded measurements at $110^{\circ}$ knee joint angle.

In the current study, HEMD ${ }_{\max }$ was found to be substantially greater than $\mathrm{QEMD}_{\max }(44.0$ vs $22.6 \mathrm{~ms})$. Although previous studies of the each muscle group in different cohorts have suggested this may be the case $(21,28-30)$, the current investigation was the first to compare the two muscle groups in the same cohort and confirmed our hypothesis. Furthermore, the delayed onset of hamstrings force production by, on average, $21 \mathrm{~ms}$ relative to the quadriceps appears to explain the lower ratio of explosive $\mathrm{H} / \mathrm{Q}$ force found in the first $50 \mathrm{~ms}$ of activation. This suggestion is supported by two pieces of evidence. First, there was strong negative relationship between the $\mathrm{HEMD}_{\text {max }}$ and the explosive H/Q force ratio at $50 \mathrm{~ms}$, indicating that individuals with a long $\mathrm{HEMD}_{\max }$ exhibited a low explosive $\mathrm{H} / \mathrm{Q}$ force ratio in this early phase of activation. Second, when the influence of EMD was factored out by comparing the explosive force of the two muscle groups from their respective force onsets, the hamstrings actually produced greater absolute force than the quadriceps at $25 \mathrm{~ms}(39 \%)$ and achieved a greater proportion of its MVF throughout the first $150 \mathrm{~ms}$ of contraction. This demonstrates that after the longer EMD of the hamstrings muscle group, it is then able to use the available force generating capacity more effectively than the quadriceps.
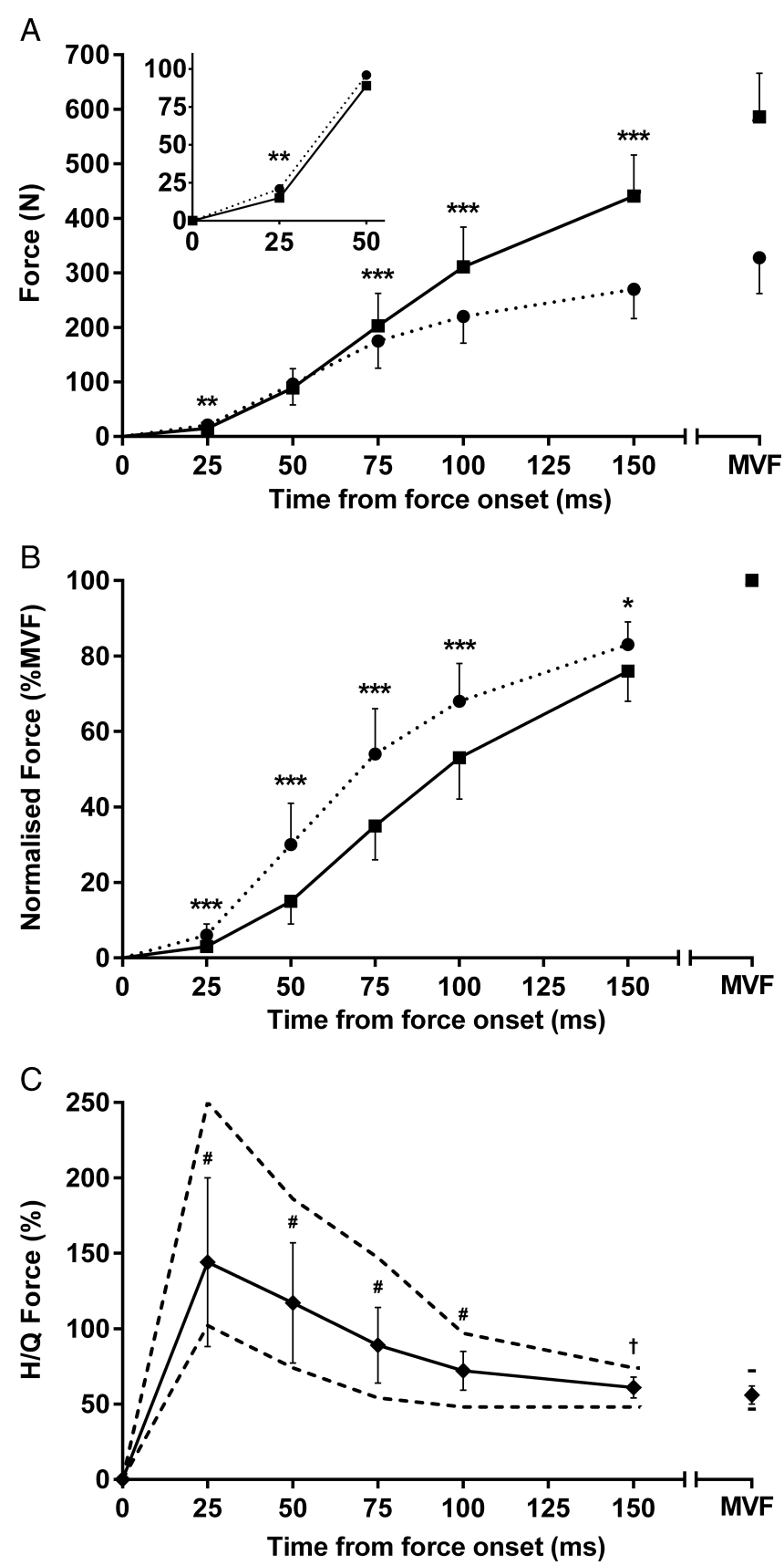

FIGURE 6-Absolute (A) and normalized (B) force of the hamstrings (closed circles) and quadriceps (closed squares) and the $H / Q$ force ratio (C) measured from force onset during the explosive efforts and at maximum voluntary force (MVF). C. Minimum and maximum values for individuals within the group with dashed lines. Data are mean \pm SD. Significant differences between muscle groups denoted by $* P<0.05$, $* * P<0.01$, and $* * * P<0.001$. Differences in explosive $H / Q$ force ratio compared with H/Q MVF denoted by ${ }^{\sharp} P<0.001$ and ${ }^{\dagger} P<0.05$.

Furthermore, when assessed from force onset the explosive $\mathrm{H} / \mathrm{Q}$ force ratio exceeded $\mathrm{H} / \mathrm{Q} \mathrm{MVF}$ at all times between 25 and $150 \mathrm{~ms}(61 \%-144 \%)$. Thus, the impaired explosive $\mathrm{H} / \mathrm{Q}$ force ratio in the first $50 \mathrm{~ms}$ from simultaneous activation does not appear to be explained by a lower capability for rapidly increasing hamstring force once initiated, rather it is a consequence of the prolonged $\mathrm{HEMD}_{\text {max }}$. 
The longer EMD found for the hamstrings compared with the quadriceps was not due to differences in agonist neuromuscular activation of the muscles, which we showed for the first time was similar in the first $100 \mathrm{~ms}$ of explosive contractions. EMD is considered to be dependent on the time taken for muscle shortening to remove slack within the series elastic component (SEC) of the muscle tendon unit (MTU [31]). In comparison to the quadriceps, the hamstrings MTU appears to have a greater absolute total tendon length, 21.728.4 vs $3.0-12.4 \mathrm{~cm}$, and relative total tendon length, $36 \%$ $42 \%$ vs $8 \%-27 \%$ MTU length $(23,32)$, largely due to substantial tendons at both ends of the hamstrings, which is not the case for the uniarticular vastii muscles within the quadriceps. This greater SEC, and thus likely greater slack, within the hamstrings appears to be the most likely explanation for its longer EMD. Muscle composition is also considered to influence EMD (40), whereby a greater proportion of Type II fibers would be expected to result in faster shortening of the muscle and removal of SEC slack. There is some evidence that the quadriceps may have a $17 \%$ greater Type II fiber percentage than the hamstrings $(15,38,45)$ that could also contribute to the shorter QEMD $_{\max }$ than $\mathrm{HEMD}_{\max }$.

Within the same muscle, shorter MTU lengths are associated with a prolonged EMD (31) because of greater slack at short lengths. The extended knee joint position $\left(150^{\circ}\right)$ for flexion and extension force measurements in the current investigation was chosen so that these reciprocal measurements were both recorded at the same extended knee joint position where injuries occur (26). A potential confounding factor in the present study was the adoption of different hip joint angles for evaluations of quadriceps and hamstrings neuromuscular performance $\left(140^{\circ}\right.$ vs $\left.180^{\circ}\right)$ due to a constraint within our knee dynamometer. However, hamstrings and quadriceps forces and thus $\mathrm{H} / \mathrm{Q}$ ratios are known to be much more sensitive to changes in knee than hip angle (36). Furthermore, despite this discrepancy in hip joint angles, the overall joint configurations used in this study would have placed the knee flexors within $10^{\circ}$ of the angle of peak knee flexor force $(6,49)$ compared with the knee extensors being $>30^{\circ}$ from the angle of peak knee extensor force $(7,48)$. Therefore, the hamstrings were assessed at a relatively long length compared with the quadriceps and closer to their optimum angle for force production $(24,36)$. This would be expected to favor a relatively short hamstrings EMD in comparison with quadriceps, yet hamstrings EMD was still almost two times greater. Therefore, it seems likely that differences in hamstrings muscle morphology, that is, longer SEC and potentially muscle composition, account for the longer EMD than the quadriceps.

Sports medicine implications. The low ratio of explosive H/Q force we have found in the early phase of activation may have important implications for dynamic knee joint stabilization and injury. The results suggest that, under certain circumstances, the hamstrings may not able to counteract the potentially harmful loading of the ACL induced by quadriceps contraction during the early phase of simultaneous activation. Although dynamic athletic activities would not typically be expected to involve precisely simultaneous and equivalent activation of opposing muscle groups, the low explosive $\mathrm{H} / \mathrm{Q}$ force ratio during the early phase of activation that we have observed may reflect an inherent functional weakness that exacerbates injury risk.

However, it is important to acknowledge that we evaluated explosive neuromuscular performance in a controlled isometric situation and from a resting state. Dynamic knee joint stabilization is likely to involve reciprocal concentric and eccentric explosive force production, and these different types of contraction have been found to influence the ability to express the available force generating capacity (43). Furthermore, both the quadriceps and hamstring muscles are usually preactivated before ground contact during dynamic athletic activities $(19,27)$, and the extent to which the hamstrings are recruited to counteract knee extensor torque, anterior tibial shear, and thus ACL loading is uncertain (25). Nevertheless, the intrinsic relative disadvantage of the hamstrings for explosive force production during the first $50 \mathrm{~ms}$ of activation would still appear to be of major functional significance.

The interindividual variation in the explosive $\mathrm{H} / \mathrm{Q}$ force ratio was very high in the first $50 \mathrm{~ms}$ of activation $(\mathrm{CV}$, $98 \%$ ) by comparison with H/Q MVF (CV, 11\%). This wide variability in explosive H/Q force found in an untrained male population may indicate large differences between individuals in the capacity for rapid muscular stabilization of the knee and further study of interindividual variability may help the evaluation of differences in injury risk. The present study was deliberately delimited to include only untrained male participants to highlight any inherent differences in the explosive neuromuscular performance of the hamstrings versus quadriceps. That is, we attempted to minimize any additional differences or variation that may be introduced by adaptations brought about by exposure to specific sports training and competition (5) and fluctuations in the menstrual cycle or contraceptive use in females $(9,33,35)$. Although the current data show a substantial depression of early phase explosive H/Q force ratio, which may reflect intrinsic human characteristics, how these findings relate to female or athletic populations remains unknown. Therefore, future investigations should compare the explosive $\mathrm{H} / \mathrm{Q}$ force ratio of males versus females because females represent a high risk population for ACL injury $(20,34)$ and evaluate the same ratio in athletes involved in high risk sports such as soccer and basketball (34)

In conclusion, the present study found that the ratio of explosive H/Q force was substantially lower in the first $50 \mathrm{~ms}$ of activation in comparison with the more traditionally reported index of muscle balance based on muscle strength, that is, H/Q MVF. The delayed production of hamstrings explosive force relative to the quadriceps, which appears to be a consequence of the prolonged hamstrings EMD, may predispose the knee joint to instability and anterior tibial translation in the period where ACL injuries are thought to occur. The wide-ranging variability in explosive H/Q force 
observed in the early phase of activation implies that the capacity for dynamic joint stabilization within a homogeneous group of untrained individuals may vary considerably and could be an important intrinsic risk factor for injury.

\section{REFERENCES}

1. Aagaard P, Simonsen EB, Andersen JL, Magnusson P, DyhrePoulsen P. Increased rate of force development and neural drive of human skeletal muscle following resistance training. J Appl Physiol. 2002;93:1318-26.

2. Aagaard P, Simonsen EB, Magnusson SP, Larsson B, Dyhre-Poulsen P. A new concept for isokinetic hamstring: quadriceps muscle strength ratio. Am J Sports Med. 1998;26:231-7.

3. Aagaard P, Simonsen EB, Trolle M, Bangsbo J, Klausen K. Isokinetic hamstring/quadriceps strength ratio: influence from joint angular velocity, gravity correction and contraction mode. Acta Physiol Scand. 1995;154:421-7.

4. Allison GT. Trunk muscle onset detection technique for EMG signals with ECG artefact. J. Electromyogr Kinesiol. 2003;13: 209-16.

5. Andrade MS, De Lira CA, Koffes FC, Mascarin NC, BeneditoSilva AA, Da Silva AC. Isokinetic hamstrings-to-quadriceps peak torque ratio: the influence of sport modality, gender, and angular velocity. J Sports Sci. 2012;30:547-53.

6. Ayala F, De Ste CM, Sainz De BP, Santonja F. Acute effects of static and dynamic stretching on hamstring eccentric isokinetic strength and unilateral hamstring to quadriceps strength ratios. J Sports Sci. 2013;31:831-9.

7. Bohannon RW, Gajdosik RL, LeVeau BF. Isokinetic knee flexion and extension torque in the upright sitting and semireclined sitting positions. Phys Ther. 1986;66:1083-6.

8. Bojsen-Moller J, Larsson B, Magnusson SP, Aagaard P. Yacht type and crew-specific differences in anthropometric, aerobic capacity, and muscle strength parameters among international Olympic class sailors. J Sports Sci. 2007;25:1117-28.

9. Bryant AL, Creaby MW, Newton RU, Steele JR. Dynamic restraint capacity of the hamstring muscles has important functional implications after anterior cruciate ligament injury and anterior cruciate ligament reconstruction. Arch Phys Med Rehabil. 2008;89: 2324-31

10. Buckthorpe MW, Hannah R, Pain TG, Folland JP. Reliability of neuromuscular measurements during explosive isometric contractions, with special reference to electromyography normalization techniques. Muscle Nerve. 2012;46:566-76.

11. Butler DL, Noyes FR, Grood ES. Ligamentous restraints to anteriorposterior drawer in the human knee. A biomechanical study. J Bone Joint Surg Am. 1980;62:259-70.

12. Cavanagh PR, Komi PV. Electromechanical delay in human skeletal muscle under concentric and eccentric contractions. Eur J Appl Physiol Occup Physiol. 1979;42:159-63.

13. Craig CL, Marshall AL, Sjostrom M, et al. International physical activity questionnaire: 12-country reliability and validity. Med Sci Sports Exerc. 2003;35(8):1381-95.

14. Croisier JL, Ganteaume S, Binet J, Genty M, Ferret JM. Strength imbalances and prevention of hamstring injury in professional soccer players: a prospective study. Am J Sports Med. 2008;36: 1469-75.

15. Dahmane R, Djordjevic S, Smerdu V. Adaptive potential of human biceps femoris muscle demonstrated by histochemical, immunohistochemical and mechanomyographical methods. Med Biol Eng Comput. 2006;44:999-1006.

16. de Ruiter CJ, Kooistra RD, Paalman MI, de HA. Initial phase of maximal voluntary and electrically stimulated knee extension torque development at different knee angles. J Appl Physiol. 2004;97: 1693-701.
This study was funded by the Nottingham Trent University and Loughborough University. The authors report no conflicts of interest.

The results of the present study do not constitute endorsement by the American College of Sports Medicine.

17. DeMorat G, Weinhold P, Blackburn T, Chudik S, Garrett W. Aggressive quadriceps loading can induce noncontact anterior cruciate ligament injury. Am J Sports Med. 2004;32:477-83.

18. Folland JP, Buckthorpe MW, Hannah R. Human capacity for explosive force production: neural and contractile determinants. Scand J Med Sci Sports. doi: 10.1111/sms.12131.

19. Gehring D, Melnyk M, Gollhofer A. Gender and fatigue have influence on knee joint control strategies during landing. Clin Biomech (Bristol, Avon). 2009;24:82-7.

20. Griffin LY, Albohm MJ, Arendt EA, et al. Understanding and preventing noncontact anterior cruciate ligament injuries: a review of the Hunt Valley II meeting, January 2005. Am J Sports Med. 2006;34:1512-32.

21. Hannah R, Minshull C, Folland JP. Whole-body vibration does not influence knee joint neuromuscular function or proprioception. Scand J Med Sci Sports. 2013;23:96-104.

22. Hodges PW, Bui BH. A comparison of computer-based methods for the determination of onset of muscle contraction using electromyography. Electroencephalogr Clin Neurophysiol. 1996;101:511-9.

23. Kellis E, Galanis N, Natsis K, Kapetanos G. Validity of architectural properties of the hamstring muscles: correlation of ultrasound findings with cadaveric dissection. $J$ Biomech. 2009;42:2549-54.

24. Kellis E, Katis A. Quantification of functional knee flexor to extensor moment ratio using isokinetics and electromyography. $J$ Athl Train. 2007:42:477-85.

25. Kingma I, Aalbersberg S, van Dieen JH. Are hamstrings activated to counteract shear forces during isometric knee extension efforts in healthy subjects? J Electromyogr Kinesiol. 2004;14:307-15.

26. Krosshaug T, Slauterbeck JR, Engebretsen L, Bahr R. Biomechanical analysis of anterior cruciate ligament injury mechanisms: three-dimensional motion reconstruction from video sequences. Scand J Med Sci Sports. 2007;17:508-19.

27. Malinzak RA, Colby SM, Kirkendall DT, Yu B, Garrett WE. A comparison of knee joint motion patterns between men and women in selected athletic tasks. Clin Biomech (Bristol, Avon). 2001;16: $438-45$.

28. Minshull C, Gleeson N, Walters-Edwards M, Eston R, Rees D. Effects of acute fatigue on the volitional and magnetically-evoked electromechanical delay of the knee flexors in males and females. Eur J Appl Physiol 2007;100:469-78.

29. Minshull C, Gleeson NP, Eston RG, Bailey A, Rees D. Single measurement reliability and reproducibility of volitional and magnetically-evoked indices of neuromuscular performance in adults. J Electromyogr Kinesiol. 2009;19:1013-23.

30. Minshull C, Rees D, Gleeson NP. Joint angle affects volitional and magnetically-evoked neuromuscular performance differentially. J Electromyogr Kinesiol. 2011;21:672-7.

31. Muraoka T, Muramatsu T, Fukunaga T, Kanehisa H. Influence of tendon slack on electromechanical delay in the human medial gastrocnemius in vivo. J Appl Physiol. 2004;96:540-4.

32. O'Brien TD, Reeves ND, Baltzopoulos V, Jones DA, Maganaris $\mathrm{CN}$. Muscle-tendon structure and dimensions in adults and children. $J$ Anat. 2010;216:631-42.

33. Phillips SK, Sanderson AG, Birch K, Bruce SA, Woledge RC. Changes in maximal voluntary force of human adductor pollicis muscle during the menstrual cycle. J Physiol. 1996;496(Pt 2):551-7.

34. Renstrom P, Ljungqvist A, Arendt E, et al. Non-contact ACL injuries in female athletes: an International Olympic Committee current concepts statement. Br J Sports Med. 2008;42:394-412. 
35. Sarwar R, Niclos BB, Rutherford OM. Changes in muscle strength, relaxation rate and fatiguability during the human menstrual cycle. J Physiol. 1996;493(Pt 1):267-72.

36. Savelberg HH, Meijer K. The effect of age and joint angle on the proportionality of extensor and flexor strength at the knee joint. J Gerontol A Biol Sci Med Sci. 2004;59:1120-8.

37. Shimokochi Y, Shultz SJ. Mechanisms of noncontact anterior cruciate ligament injury. J Athl Train. 2008;43:396-408.

38. Staron RS, Hagerman FC, Hikida RS, et al. Fiber type composition of the vastus lateralis muscle of young men and women. J Histochem Cytochem. 2000;48:623-9.

39. Staude DH. Precise onset detection of human motor responses using a whitening filter and the log-likelihood-ratio test. IEEE Trans Biomed Eng. 2001;48:1292-1305.

40. Taylor AD, Humphries B, Smith P, Bronks R. Electrophoretic separation of myosin heavy chain isoforms in the human $\mathrm{m}$. vastus lateralis: references to reproducibility and relationships with force electromechanical delay, fibre conduction velocity, endurance and electromyography. Arch Physiol Biochem. 1997;105:10-8.

41. Thorstensson A, Karlsson J, Viitasalo JH, Luhtanen P, Komi PV. Effect of strength training on EMG of human skeletal muscle. Acta Physiol Scand. 1976;98:232-6.

42. Tillin NA, Jimenez-Reyes P, Pain MT, Folland JP. Neuromuscular performance of explosive power athletes versus untrained individuals. Med Sci Sports Exerc. 2010;42(4):781-90.

43. Tillin NA, Pain MT, Folland JP. Contraction type influences the human ability to use the available torque capacity of skeletal muscle during explosive efforts. Proc Biol Sci. 2012;279:2106-15.
44. Tillin NA, Pain MT, Folland JP. Identification of contraction onset during explosive contractions. Response to Thompson et al. "Consistency of rapid muscle force characteristics: influence of muscle contraction onset detection methodology" [J Electromyogr Kinesiol 2012;22(6):893-900]. J Electromyogr Kinesiol. 2013;23: $991-4$.

45. Tirrell TF, Cook MS, Carr JA, Lin E, Ward SR, Lieber RL. Human skeletal muscle biochemical diversity. J Exp Biol. 2012; 215:2551-9.

46. Tsaopoulos DE, Baltzopoulos V, Richards PJ, Maganaris CN. In vivo changes in the human patellar tendon moment arm length with different modes and intensities of muscle contraction. J Biomech. 2007;40:3325-32.

47. Ward SR, Eng CM, Smallwood LH, Lieber RL. Are current measurements of lower extremity muscle architecture accurate? Clin Orthop Relat Res. 2009;467:1074-82.

48. Wong PW, van Haselen J. Revisiting the influence of hip and knee angles on quadriceps excitation measured by surface electromyography. International SportMed Journal. 2010;11: 313-23.

49. Worrell TW, Karst G, Adamczyk D, et al. Influence of joint position on electromyographic and torque generation during maximal voluntary isometric contractions of the hamstrings and gluteus maximus muscles. J Orthop Sports Phys Ther. 2001;31: 730-40.

50. Zebis MK, Andersen LL, Ellingsgaard H, Aagaard P. Rapid hamstring/quadriceps force capacity in male vs. female elite soccer players. J Strength Cond Res. 2011;25:1989-93. 